\title{
Prototipo generador de energía "AKS" como una idea emprendedora en Santa Rosalía, Baja California Sur
}

\section{Prototype energy generator "AKS" as an entrepreneurial idea in Santa Rosalía, Baja California Sur}

HERNÁNDEZ-VALENZUELA, Juan Carlos $\dagger^{*}$, MEZA-ARELLANO, Antonio, GÓMEZ-MAYORAL, Karime Belmaris y TOBA-AGUIRRE, Jesús Ángel

Tecnológico Superior de Mulegé. Centro, Loma los Frailes S/N, Centro, 23920 Santa Rosalía, B.C.S.

ID $1^{\text {er }}$ Autor: Juan Carlos, Hernández-Valenzuela / ORC ID: 0000-0003-3523-232, Researcher ID Thomson: P-4344-2018, CVU CONACYT ID: 278735

ID $1^{\text {er }}$ Coautor: Antonio, Meza-Arellano / ORC ID: 0000-0002-6553-8392, Researcher ID Thomson: P-6194-2018, CVU CONACYT ID: 404918

ID $2^{\text {do }}$ Coautor: Karime Belmaris, Gómez-Mayoral

ID $3^{\text {er }}$ Coautor: Jesús Ángel, Toba-Aguirre

DOI: $10.35429 / J C A .2020 .14 .4 .8 .19$

Recibido Marzo 30, 2020; Aceptado Junio 25, 2020

\section{Resumen}

El presente artículo muestra los resultados obtenidos del estudio de mercado que se llevó a cabo en la comunidad de Santa Rosalía, Baja California Sur, con el fin de conocer los gustos, preferencias y opiniones de los consumidores sobre el prototipo generador de energía "AKS", el cual es una bicicleta estática que se encuentra conectada a una pila de 12 volts y al pedalear en ella, se va generando energía por medio de un motor generador, de tal manera que todo lo producido se va almacenando en la pila y de ahí con la ayuda de un inversor se saca la corriente, con la intención de reducir el consumo de energía eléctrica, lo que se paga por ella y la contaminación en el medio ambiente. donde se utilizó una investigación exploratoria-descriptiva, para poder obtener los resultados, analizarlos y luego llevarlos a cabo para la mejora del prototipo, todo esto a través de diferentes herramientas, tales como, encuesta, entrevistas a los establecimientos y observación directa. De modo que con los resultados obtenidos se lograron ver las respuestas del cómo crear un producto que cumpla totalmente con sus expectativas y que consiga generar grandes ventas y obtener un buen posicionamiento en el mercado.

Prototipo, Generar, Energía, Bicicleta, Necesidades, Consumo

\begin{abstract}
This article shows the results obtained from the market study that was carried out in the community of Santa Rosalia, Baja California Sur, in order to know the tastes, preferences and opinions of consumers about the "AKS" energy generating prototype, which is an exercise bike that is connected to a 12volt battery and when pedaling on it, energy is generated by means of a generator motor, so that everything produced is stored in the battery and from there with the help of an inverter the current is taken out, with the intention of reducing the consumption of electric energy, what is paid for it and pollution in the environment. where an exploratory-descriptive investigation was used, in order to obtain the results, analyze them and then carry them out for the improvement of the prototype, all this through different tools, such as, survey, interviews to establishments and direct observation. So with the results obtained, they were able to see the answers of how to create a product that fully meets their expectations and that manages to generate large sales and obtain a good market positioning.
\end{abstract}

Prototype, Generate, Energy, Bicycle, Needs, Consumption

Citación: HERNÁNDEZ-VALENZUELA, Juan Carlos, MEZA-ARELLANO, Antonio, GÓMEZ-MAYORAL, Karime Belmaris y TOBA-AGUIRRE, Jesús Ángel. Prototipo generador de energía "AKS" como una idea emprendedora en Santa Rosalía, Baja California Sur. Revista de Cómputo Aplicado. 2020. 4-14: 8-19.

\footnotetext{
* Correspondencia al Autor (Correo Electrónico: Carlos.hernandez@itesme.edu.mx)

$\dagger$ Investigador contribuyendo como primer autor.
} 


\section{Introducción}

Durante los últimos años se ha notado el crecimiento del consumo de la energía eléctrica, debido a que la tecnología va cubriendo las necesidades de cada una de ellas y la mayoría de los productos que se van dando a conocer se utilizan con base a este proceso, por esa razón las personas están muy acostumbradas a la comodidad que brinda la electricidad ya que gracias a ella funcionan distintos aparatos que normalmente se utilizan en el trabajo y el hogar. Cabe destacar que la energía eléctrica es muy importante para muchas cosas pero así como tiene sus ventajas también sus desventajas, como por ejemplo: daña al medio ambiente debido a la forma en la que se produce y al utilizarse se debe pagar, lo cual es muy complicado para algunas personas, ya que no todos cuentan con el recurso económico suficiente como para poder mantenerse al margen y cumplir esa necesidad, es por ello que nació la idea de crear un prototipo generador de energías limpias, el cual tiene el propósito de encontrar un nuevo sustento a través de un sistema encargado de poner en práctica las energías renovables, porque hoy en día son una gran relevancia para la sociedad ya que en años anteriores no se conocía lo que en realidad era y los beneficios que esta aporta, como por ejemplo: el poder de encender aparatos eléctricos que normalmente se utilizan en el hogar y en áreas de trabajo, además los beneficios que tiene el prototipo ocurren sin dañar el medio ambiente y sin consumir tanto la energía eléctrica, de tal manera que se hace una gran aportación al disminuir notoriamente los gastos que se generan por ella misma y se ahorra una parte del recurso económico.

Sin embargo, es necesario conocer acerca de los consumidores y hacia quien debe ser dirigido, debido a que ellos son parte de lo más importante dentro del mercado ya que se deben de cumplir con sus expectativas para que de esa manera se interesen por el producto y logren comprarlo. Por esta razón la importancia de realizar un estudio de mercado, ya que en él se permite conocer los distintos gustos y preferencias que tiene el consumidor, con las herramientas utilizadas para la obtención de resultados, como lo son, encuesta, entrevistas a establecimientos y observación directa, con el fin de obtener información más específica de parte de los consumidores y plasmarlos en una gráfica para representarlos de una manera más fácil y entendible.

\section{Metodología}

El método utilizado fue una investigación exploratoria y descriptiva, se realizaron encuestas las cuales se aplicaron a 6 diferentes colonias de la localidad de Santa Rosalía, Baja California Sur, esto se hizo con el fin de obtener distintos puntos de vista y conocer más acerca de los posibles consumidores, claro respecto al prototipo generador de energía, es por ello que para determinar la muestra de población se acudió a la página del Instituto Nacional de Estadística y Geografía, y de acuerdo con (INEGI, 2015) existen 14,160 habitantes, posteriormente se sacó el resultado de la anterior cantidad en una muestra, con base a la fórmula que respecta a la muestra poblacional, la cual es la siguiente (Tapia, 2012):

$n=\frac{N \sigma^{2} Z^{2}}{(N-1) e^{2}+\sigma^{2} Z^{2}}$

En la formula $n$ es igual al tamaño de la muestra, mientras que $\mathrm{N}$ es el tamaño de la poblacion, $\sigma$ es desviación estándar de la población que, generalmente cuando no se tiene su valor, suele utilizarse un valor constante de $0,5, \mathrm{Z}$ es obtenido mediante niveles de confianza. Este es un valor constante que, si no se tiene su valor, se lo toma en relación al $95 \%$ de confianza equivale a 1,96 (como más usual) o en relación al 99\% de confianza equivale 2,58, valor que queda a criterio del encuestador y e es el límite aceptable de error muestral que, generalmente cuando no se tiene su valor, suele utilizarse un valor que varía entre el $1 \%(0,01)$ y $9 \%(0,09)$, valor que queda a criterio del encuestador, esto arrojo como resultado un total de 374 , sienta esta la determinacion aleaotoria a la que fue dirigida la encuesta.

$\frac{(14.160)(0.5)^{2}(1.96)^{2}}{(14.160-1)(0.05)^{2}+(0.5)^{2}(1.96)^{2}}=\frac{13,599.264}{36.3579}=374$

En este método se encuesto a 62 personas entre 6 colonias en la localidad, las cuales llevan por nombre: Nopalera, Nueva Santa Rosalía, Magisterial, Centro, Cuauhtémoc y Costa azul.

Otra de las herramientas que se utilizó para la investigación de mercado, fue la observación directa, la cual proporciona información cualitativa y cuantitativa sobre el producto, en esta se pudo observar las reacciones que tuvieron las personas al observar y conocer del prototipo "AKS".

HERNÁNDEZ-VALENZUELA, Juan Carlos, MEZAARELLANO, Antonio, GÓMEZ-MAYORAL, Karime Belmaris y TOBA-AGUIRRE, Jesús Ángel. Prototipo generador de energía "AKS" como una idea emprendedora en Santa Rosalía, Baja California Sur. Revista de Cómputo Aplicado. 2020 
Para desarrollar esta observación, se realizó la misma fórmula que se utilizó en la herramienta anterior, solo que con los siguientes datos:

$\mathrm{N}=14,169$

$\sigma=0.5$

$\mathrm{Z}=1.90$

$\mathrm{e}=0.5$

$\frac{(14.160)(0.5)^{2}(1.90)^{2}}{(14.160-1)(0.10)^{2}+(0.5)^{2}(1.90)^{2}}=\frac{12,779.4}{142.4925}=90$

Este método se les hizo a 90 personas, de manera en que se colocó el prototipo generador acompañado de una mesa con un mantel y una computadora, en ella se mostró el logotipo y se dieron a conocer los diferentes materiales, características y funciones del producto, de igual manera se realizó una escala de medición con 8 incentivos y se le aplicó a cada una de las personas interesadas, esto se llevó acabo en la cancha Pedro Rucho Ceseña, el día 20 de marzo de 2019.

\section{Resultados obtenidos}

\section{Perfil socioeconómico del consumidor}

La muestra estuvo integrada por 374 habitantes que es un equivalente de $100 \%$ en todos los rubros. Dentro de las encuestas realizadas el $53 \%$ de ellos fueron hombres y el $47 \%$ restante eran mujeres, mientras que el $63 \%$ de ellos están en matrimonio y el $37 \%$ sobrante es soltero. La edad promedio de los encuestados se encuentra en el rango de 30-39, representando el $31 \%$ de la muestra, lo que se considera un estado de población media ya que no son ni muy jóvenes y tampoco muy adultos (de la tercera edad). Con relación a la escolaridad, se encontró que el $45 \%$ termino su estudio en bachillerato; $35 \%$ licenciatura; $13 \%$ secundaria; $6 \%$ primaria; $1 \%$ no estudio y un $0 \%$ en postgrado.

Por otro lado, los resultados muestran que el porcentaje más alto del número de habitantes en el hogar es de 3 con un $32 \%$, seguido con un $26 \%$ en familias de $3 ; 18 \%$ de 1 $2 ; 17 \%$ de 5 y $7 \%$ de 6 o más. Otro rubro importante es el tipo de consumidores por nivel de ingreso mensual familiar, alrededor del $33 \%$ tiene ingresos entre \$8,221 y \$16,440; el $24 \%$ de los hogares tienen ingresos mensuales que oscilan entre $\$ 4,988$ a $\$ 8,220$; el $20 \%$ tiene entre $\$ 16,441$ o más; y resto tiene menores ingresos (cifras en pesos mexicanos).

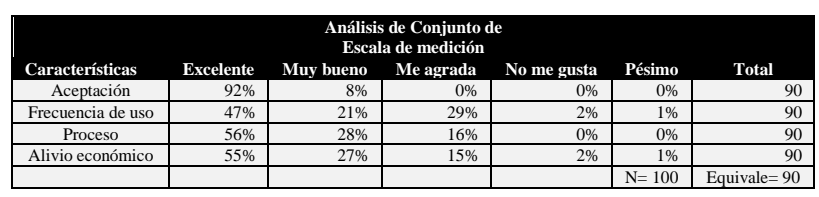

Tabla 1 Análisis de conjunto del prototipo Fuente: elaboración propia; tomada del estudio: Análisis de las preferencias del consumidor a través de la observación directa

De acuerdo con Villavicencio J. (2016) el análisis de conjunto es cualquier método des composicional que estima la estructura de las preferencias de un consumidor, sin embargo, esta técnica se utilizó de una manera agradable realizando una escala de medición usando la fórmula del muestreo poblacional, a una muestra de 90 personas de la localidad de Santa Rosalía, Baja California Sur, esto se hizo con el fin de conocer cuáles son los gustos y preferencias de los consumidores. De tal manera que para iniciar con la escala el prototipo generador de energía "AKS" se colocó a las afueras de la cancha Pedro Rucho Ceseña y se dieron demostraciones acerca de los resultados que se pueden obtener con el prototipo para que las personas que transitaran por el lugar lograran ver sus funciones y beneficios. Después de las pruebas e información que se brindó durante la escala de medición también se tomaron en cuenta algunas preguntas para saber si el producto lograría tener un buen posicionamiento en el mercado, si el proceso es el adecuado o sería mejor tener algunos cambios respecto a él, y si en verdad lograría tener un alivio económicoDonde se puede observar en la tabla 1 los resultados obtenidos, muestran un $92 \%$ de aceptación hacia el producto y al otro $8 \%$ le pareció muy bueno, así mismo comentaron la frecuencia de uso que le darían a este, dando más variación en los resultados ya que el $47 \%$ comentaba que les parecía excelente, el $21 \%$ decía que era muy bueno, mientras que el $29 \%$ pensaba que era agradable $y$ a un $3 \%$ no les parecía tan interesante y algo pésimo. Con respecto al proceso que se realiza para generar energía, no se encontraron malos comentarios los cuales hicieran tomar un cambio en el, ya que el $56 \%$ dijo que es excelente, el $28 \%$ lo cree muy bueno $\mathrm{y}$ al otro $16 \%$ le agrado. 
Al terminar la escala de medición se realizó una pregunta hacia las personas que se acercaban y se tomaban el tiempo en observar el prototipo, en la cual se preguntaba si creían que lo realizado podría lograr tener impacto y un alivio económico, en donde la mayoría respondió que sí, con porcentajes de $55 \%$ excelente, $27 \%$ muy bueno, $15 \%$ agradable, $2 \%$ no lo creía y $1 \%$ para nada, pésimo.

Es por ello que con los resultados obtenidos se vieron las grandes oportunidades que se podrán tener con el producto en un futuro, ya que se tuvieron muy buenos resultados y aceptación hacia él, de igual manera existirá un gran impacto ya que tiene muchos beneficios, como por ejemplo: que genera energías limpias y en comparación con la anergia eléctrica no contamina al medio ambiente, también agregando que reduce el consumo y gastos ocasionados por la energía eléctrica ya que se pueden conectar aparatos electrónicos que normalmente utilizan en el hogar o son indispensables para el hombre, solo gastando de su energía corporal y ayudando al mismo tiempo a su salud.

\section{Factor de consumo}

Durante los últimos años se ha notado el crecimiento del consumo de la energía eléctrica, debido a que la tecnología va cubriendo las necesidades de cada una de ellas y la mayoría de los productos que se van dando a conocer se utilizan con base al poder de la corriente, sin embargo, un estudio realizado por la Agencia Internacional de la Energía [AIE], (2015), expone que la demanda mundial de electricidad aumentará un $70 \%$ hasta 2040, por ello la tecnología evoluciona cada día más y ayuda a las personas a adaptarse a cualquier tipo de cambio, la cual es utilizada para un sinfín de actividades que realizan las empresas para llevar una mejor productividad u organización y así lograr mantenerla en un buen nivel y seguir con éxito.
De tal manera que la energía eléctrica es muy importante para muchas cosas, pero así como tiene sus ventajas también sus desventajas, como por ejemplo: daña al medio ambiente debido a la forma en la que esta se produce y al utilizarse se debe pagar, lo cual es muy complicado para algunas personas, ya que no todos cuentan con el recurso económico suficiente como para poder mantenerse al margen con la utilización de los aparatos electrónicos que normalmente se usan en el hogar, los cuales podrían ser: microondas, abanicos, televisión, refrigeradores, cargadores, etc. Sin embargo, en un estudio realizado en la localidad de Santa Rosalía, Baja California Sur se obtuvieron datos los cuales se muestran en el Gráfico 1, en ella se pueden dar a conocer los resultados de los pagos que se realizan por la utilización de aparatos electrónicos y la cantidad de personas en el hogar, también ilustra que un 95\% de habitantes consume solo energía eléctrica y el $4 \%$ restante no lo hace, solo energías renovables, usando aparatos como: paneles solares, luces solares y generadores eólicos. A pesar de conocer todos los problemas que ocurren al momento de generar electricidad las personas siguen con su utilización, ya que es la forma más cómoda de obtener energía y satisfacer sus necesidades rápidamente, sin embargo, el prototipo generador de energía "AKS" también hace funcionar los aparatos que normalmente se usan en el hogar, solo que de lo contrario a la energía eléctrica el proceso de este prototipo no causa ningún daño a las personas ni al medio ambiente, debido a que solo se necesita del esfuerzo humano para pedalear en una bicicleta y generar energía.

\section{Pago por el consumo de electricidad}

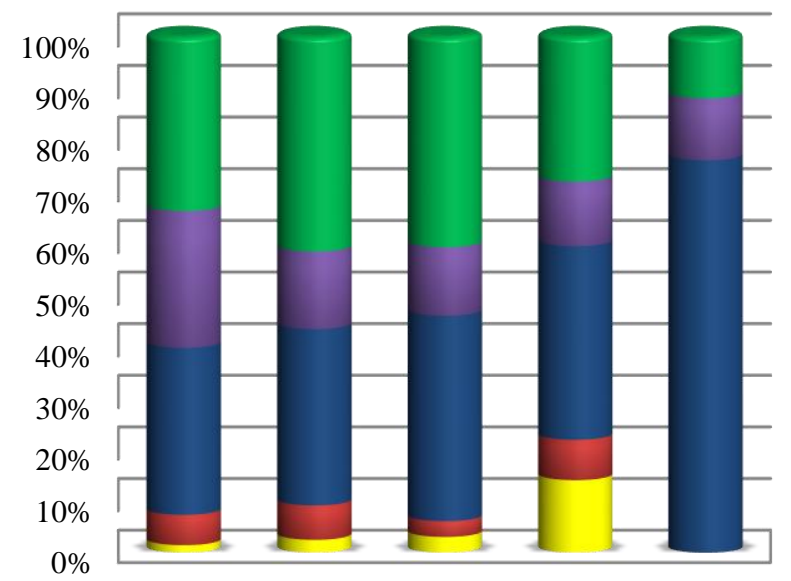

Gráfico 1 Pago por el consumo de electricidad

Fuente: elaboración propia; tomada del estudio: Análisis de estudio de mercado del "Prototipo generador de energía "AKS"

HERNÁNDEZ-VALENZUELA, Juan Carlos, MEZAARELLANO, Antonio, GÓMEZ-MAYORAL, Karime Belmaris y TOBA-AGUIRRE, Jesús Ángel. Prototipo generador de energía "AKS" como una idea emprendedora en Santa Rosalía, Baja California Sur. Revista de Cómputo Aplicado. 2020 
En verde de $\$ 600$ a $1000,35.82 \%$, en mostaza de $\$ 400$ a $600,16.04 \%$, en gris de $\$$ 1000 o más, $38.77 \%$, anaranjado de $\$ 100$ a 300, $5.34 \%$, y en amarillo no utilizan energía eléctrica, $4.27 \%$.

Este producto ha logrado tener un gran impacto en la sociedad ya que genera energía que puede ser utilizada en cualquier aparto electrónico, además de que no es necesario estar en casa para poder usarla, debido a que es de un tamaño adecuado y puede colocarse en distintos lugares.

Es decir que el prototipo generador de energía supera a los productos que se encuentran en el mercado, ya que aparte de cumplir todos los beneficios mencionados anteriormente ayuda a disminuir los gastos en el hogar, dado a que el consumo de energía eléctrica disminuye de igual forma.

\section{Medios de publicidad}

La publicidad es un factor importante dentro de las empresas ya que gracias ello se da a conocer los precios, promociones, mejoras y ofertas de un producto a la sociedad, de esa manera aumenta la posibilidad de que se generen más ventas y crezcan las ganancias. Esta desempeña un papel de gran importancia en cualquier empresa ya que esta sirve de medio para comunicar a muchas personas el mensaje de un patrocinador a través de un medio impersonal y está diseñada para convencer a una persona para que compre un producto, para apoyar a una causa o incluso para obtener menor consumo, tiene una gran influencia sobre el consumidor; a través de una buena publicidad se puede lograr vender grandes volúmenes de mercancías, todo depende de la capacidad que esta tenga para convencer al público para que compre el producto. De acuerdo con Sweezy (2015) una de las funciones de la publicidad es el reforzamiento de los deseos y preferencias de los consumidores, social y/o bióticamente determinados, por ello muestran el producto a la comunidad de manera llamativa y en un medio que la mayoría de los habitantes de la localidad logren verlo.

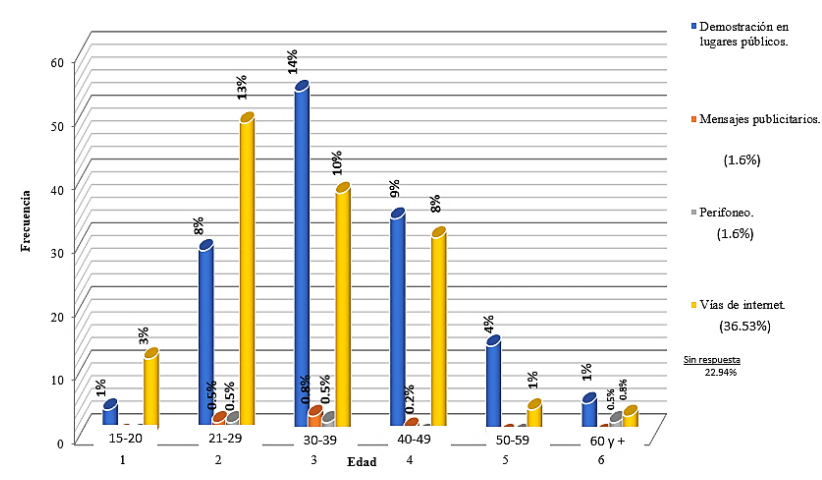

Gráfico 2 Medios de publicidad para el reconocimiento y venta del producto

Fuente: elaboración propia; tomada del estudio; Análisis del estudio de mercado del "Prototipo generador de energía $A K S^{\prime \prime}$

En el gráfico 2 se pueden apreciar los datos que se obtuvieron del estudio de mercado, los cuales indican porcentajes de los medios de publicidad que desean las personas para conocer sobre el producto y su venta.

De esta forma, el $37.33 \%$ dijeron que les gustaría conocerlo por demostraciones en lugares públicos debido a que de esa manera se podrán hacer preguntas al tener alguna duda y conocer todo acerca del prototipo, dentro del porcentaje mencionado anteriormente se encuentran otros más, los cuales definen las edades de quienes prefirieron ese medio de publicidad, como por ejemplo: de 15-20 años el $1 \%$ lo escogió, seguido de $21-29$ el $8 \%$ creyó que sería el más adecuado, después entre 30-39 se encontró la cantidad más alta, que sería de $14 \%$, para seguir con el 9\% en 40-49, un 4\% en 50-59 y terminando con el porcentaje más bajo el cual fue $0.5 \%$ en $60 \mathrm{y}+$.

Del mismo modo el $36.53 \%$ considero que lo más adecuado sería promocionarlo por vías de internet ya que la mayoría de las personas pasan gran parte de su día o están al tanto de ellas, aunque de igual manera se dan a conocer las comparaciones de preferencias por las edades, mostrando porcentajes de 3\% en 15-20, $13 \%$ en $21-29,10 \%$ dentro de $30-39$, en $40-49$ un $8 \%$ y las respuestas más bajas hacia este medio se encontraron en $50-59$ con un $1 \%$ y 60 $\mathrm{y}+$ con un $0.8 \%$ 
Seguido de un $1.6 \%$ por dos medios de publicidad, los cuales son mensajes publicitarios y perifoneo, encontrándose solo dentro de 21-29 años con un $0.5 \%$ en los dos, seguido de 30-39 mostrando un $0.8 \%$ en mensajes publicitarios y en $40-490.2 \%$, y terminando con un $0.5 \%$ en 60 $\mathrm{y}+$ prefirieron perifoneo debido a que las personas de la tercera edad no salen mucho del hogar y no utilizan tanto el internet.

Debido a los porcentajes más altos que se obtuvieron, se realizaron demostraciones en lugares públicos donde transiten muchas personas, de modo que se colocó el prototipo y se hicieron pruebas del mientras se dan a conocer sus beneficios. Aunque de igual forma se creó una página en facebook donde se subirán videos e imágenes mostrando información de él. De tal manera que una de las principales estrategias para lograr un impacto dentro de la sociedad fue crear un producto distinto a los que hoy existen en el mercado, en los cuales no se encontraba ninguno que pudiera generar energía y almacenarla al mismo tiempo.

\section{Localización de su venta}

Las ventas son un factor importante dentro de las empresas ya que gracias a ellas aumenta la productividad y así mismo se genera una mayor demanda. Figueroa (2015) comentaba que las ventas tienen como función principal comercializar los bienes o servicios que ofrece una empresa, aunque también lo que se espera de esto es recuperar el capital invertido durante la realización del producto, debido a esto la empresa llega a posicionarse en un buen nivel en el mercado para ser competente con otras organizaciones con productos similares los cuales son las placas solares, generadores eólicos, luces solares y las tradicionales planta generadoras de luz a base de gasolina.

Respecto al prototipo "AKS" se espera cumplir las metas planteadas para la efectividad de la venta, contemplando unos de los factores más importantes el cual es la localización.

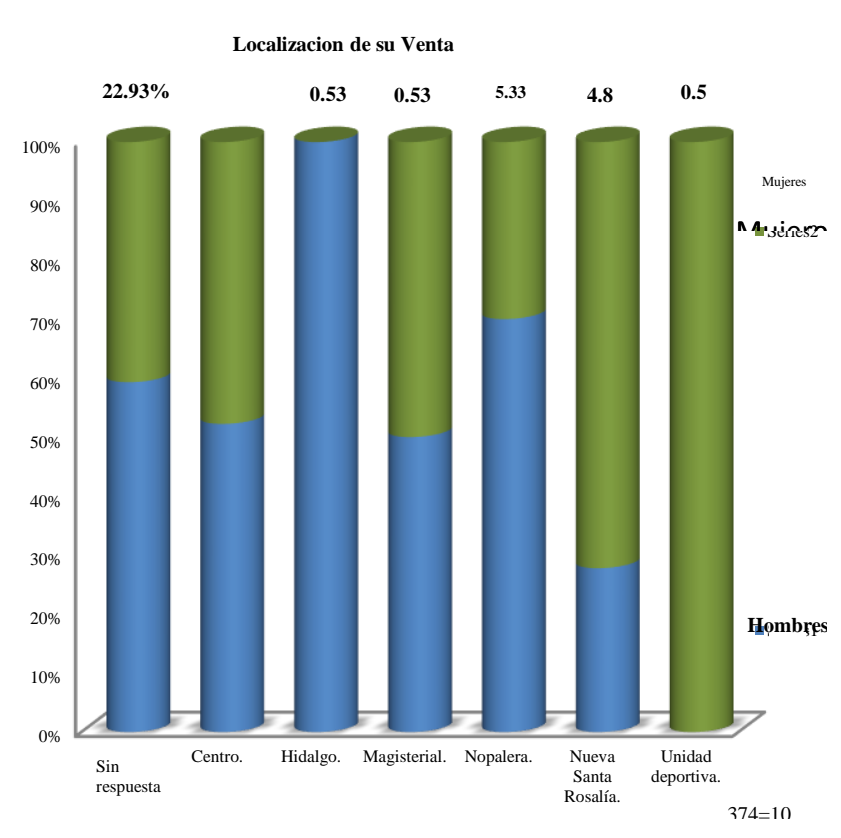

Gráfico 3 Localización de su venta

Fuente: elaboración propia; tomada del estudio; Análisis del estudio de mercado del "Prototipo generador de energía $A K S$

En el Gráfico 3, se puede mostrar que fue lo que el estudio de mercado arrojo sobre el punto de venta más conveniente que las personas de la localidad se les haría más accesible para encontrar el producto.

Como se puede observar en la gráfica un $22.93 \%$ no respondieron a la serie de preguntas solicitadas el cual es un equivalente a 86 personas de ambos sexos, ellos comentaban que no utilizarían un producto con base a las energías renovables y no dieron sus opiniones respecto a él. De igual manera un $0.53 \%$ de la parte de la localidad que es equivalente a 2 personas respondieron que la localización de este producto les gustaría que se ubicara en la colonia hidalgo y seguidamente en la colonia unidad deportiva, así mismo un $65.33 \%$ de la población equivalente a 245 personas respondieron que en la colonia centro sería la mejor ubicación del local para la venta de este producto, ya que la mayor parte de las personas se enfocan en visitar la gran variedad de establecimientos que se encuentran ahí, debido a que es la colonia con más locales de comercio de la comunidad. 
Cabe mencionar que la ubicación de la colonia centro efectivamente sería la más adecuada para la venta de este producto ya que la mayor parte de las personas que transitan por este lugar de la comunidad son aptas para utilizar este tipo de producto que se pretende poner a la venta para mejorar la vida de las personas y mejorar la economía de la empresa para seguir satisfaciendo las distintas necesidades de las personas.

\section{El interés y aceptación del consumidor hacia el proceso del prototipo "AKS"}

Debido a la contaminación, los altos pagos que se generan por la utilización de la energía eléctrica, y los problemas que ocurren durante los desastres naturales, como por ejemplo: los apagones de luz durante los huracanes, la sociedad busca otras alternativas las cuales puedan cubrir sus necesidades sin tener que pasar por ese tipo de cosas, así mismo se basan en los aparatos que funcionan gracias a las energías renovables, como lo podrían ser las plantas generadoras de luz con base a gasolina y las placas solares, que son las que se utilizan comúnmente. Ortiz J. (2015) comentaba que la contribución de las energías renovables al desarrollo económico de cualquier medio humano ayuda a mantener los equilibrios sociales.

Sin embargo, el prototipo generador de energía tiene las mismas funciones de los productos mencionados anteriormente solo que el proceso de este es diferente ya que se necesita realizar ejercicio para poder obtener sus funciones. En el Gráfico 4, se muestra cual fue el resultado de las personas que estarían dispuestas a realizarlo, los cuales se interpretaron de la siguiente manera:

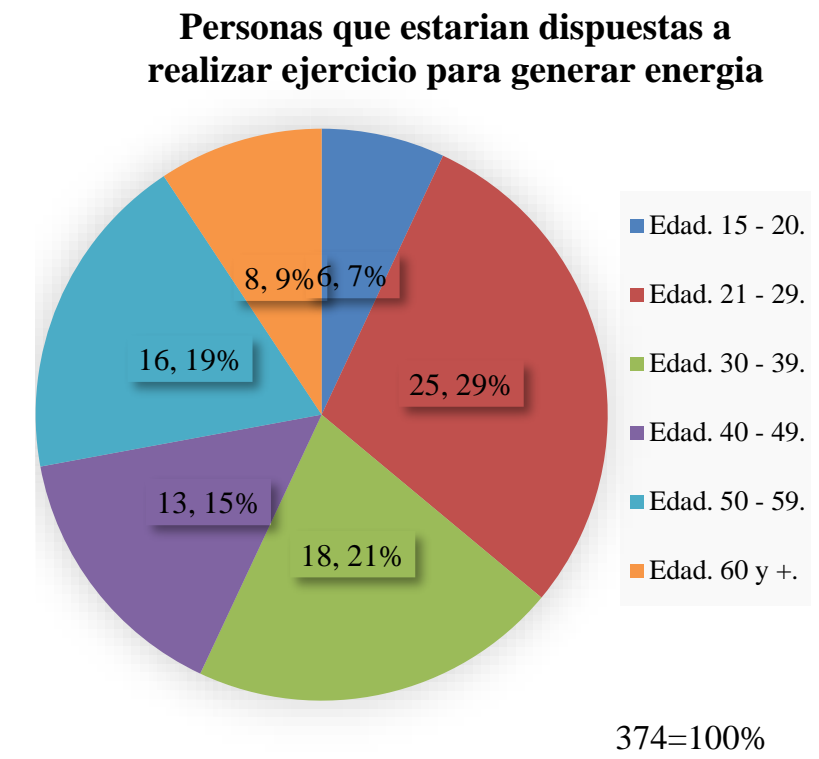

Gráfico 4 Personas que estarían dispuestas a realizar ejercicio para generar energía

Fuente: elaboración propia; tomada del estudio; Análisis del estudio de mercado del "Prototipo generador de energía $A K S^{\prime \prime}$

Se puede ver la edad de aceptación del proceso de generar energía, dando como resultado que de un $100 \%$, el $25.29 \%$ es de 21 29 años, $18.21 \%$ de 30-39 años, el $13.15 \%$ de 40-49 años, $16.19 \%$ es de $50-59$, el $8.9 \%$ es de $60 \mathrm{y}+$ años, y por último el $6.7 \%$ es de $15-20$, esto da como resultado que las personas en edades de 21 a 29 años son los que más están a favor del proceso de cómo generar energía.

Con respecto a los resultados obtenidos se pudo lograr identificar quienes serán los consumidores que demanden este producto ya que se encuentran porcentajes y respuestas muy diferentes, además de que todos adquieren distintas formas de pensar también tiene mucho que ver las edades o la salud de cada persona, como por ejemplo: los bebes, niños o cualquiera que no sepa utilizar una bicicleta no estarían interesadas en un prototipo que tuviera ese proceso, o igual personas de la tercera que ya no tienen la misma energía, arriesgan a lastimarse o se les tiene prohibido hacer muchos esfuerzos físicos por las situaciones de salud en las que se encuentren. De tal manera que el prototipo no estará dirigido directamente hacia ellos si no a quienes puedan utilizarlo y tengan esa motivación o gusto por ejercitarse, debido a que ellos no dudarían o se quejarían de realizar ejercicio para generar energía ya que se benefician de dos maneras que sería sentir estabilidad con su cuerpo y satisfacer sus necesidades con base a la energía generada y almacenada. 


\section{Preferencias de compra del consumidor}

La compra supone una operación de intercambio; la persona que compra (consumidor) entrega dinero a cambio de un bien o servicio deseado, mientras que la persona que vende (vendedor) ofrece el bien o servicio a cambio de una cantidad de dinero determinada. En la economía, los consumidores son los demandantes, mientras que los vendedores son los oferentes. De acuerdo con Sangri A. (2014) comenta que las compras es alcanzar la posesión de una cosa, por trabajo. Sinónimo de comprar, conseguir y obtener, acción de adquirir y hacer propio lo que a nadie pertenece. La importancia de esta acción es que esta actividad radica en su vitalidad para el éxito de las empresas, ya que determina la efectividad de la administración de los bienes adquiridos. Por ese motivo la empresa necesita de insumos, materia prima, componentes y refacciones para su funcionamiento, sin los cuales no podría lograr la producción y distribución de sus productos. Es por ello que la adquisición de productos en la actualidad es algo cotidiano para los habitantes, ya que día a día las personas requieren de distintos productos que están en el mercado pero que deben de dar una remuneración por obtenerlos, debido a lo ya mencionado se creó un prototipo generador de energía llamado AKS, con el fin de auxiliar a la energía eléctrica y así mismo favorecer a la comunidad en el consumo de energía.

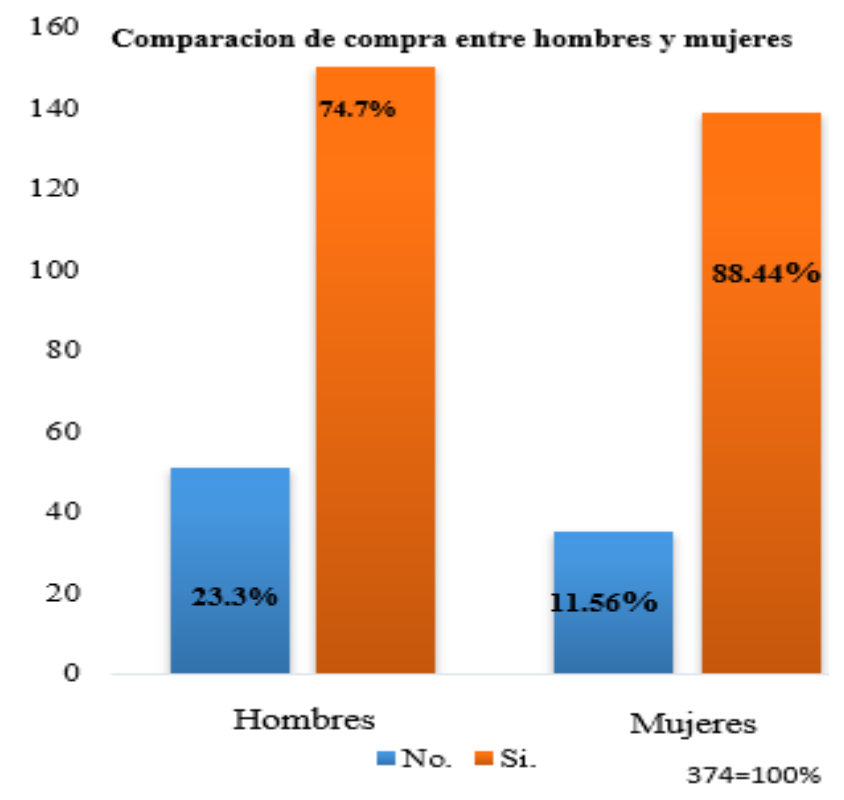

Gráfico 5 Comparación de compra entre hombres y mujeres

Fuente: elaboración propia; tomada del estudio: Análisis de estudio de mercado del "Prototipo generador de energía $A K S^{\prime \prime}$
En el Gráfico 5 se puede observar la aceptación de compra del producto entre hombres y mujeres, en donde se muestra que hay mayor probabilidad de que los mayores consumidores de este producto sean las mujeres, ya que se observa que un $88.44 \%$ contestaron que si utilizarían un prototipo generador con base a las energías renovables, en cambio los hombres dijeron que si lo utilizarían con un $74.7 \%$, estos porcentajes aclaran que los compradores más comunes de la comunidad sean mujeres.

Debido a los porcentajes obtenidos se muestra que la empresa AKS estará generando una ganancia notoria ya que con los resultados que se muestran, se dice que las ventas se dispararían en un mayor porcentaje, de tal manera que este producto sea comprado por gran parte de las mujeres que desean economizar el consumo de energía eléctrica.

\section{Análisis del precio}

El precio es el único elemento de la mezcla de mercadotecnia que produce ingresos; todos los otros elementos representan costos. De tal manera que la importancia que tiene este dentro de la economía es que se considera que la determinación de precios es la actividad clave dentro del sistema capitalista de la libre empresa. Flores H. (2016) comentaba que el precio es uno de los cuatro pilares fundamentales de la mezcla del marketing, los cuales son: producto, precio, plaza y promoción.

Es por ello que el precio del prototipo "AKS" es elevado, pero tomando en cuenta que solo se vería como un gasto al principio, es más como una inversión, ya que, al usarlo se reduce considerablemente lo que se paga por el consumo de luz eléctrica, además, debido a que el prototipo no necesita de muchos mantenimientos especiales lo vuelven un producto bastante redituable y agradadle de comprar. 


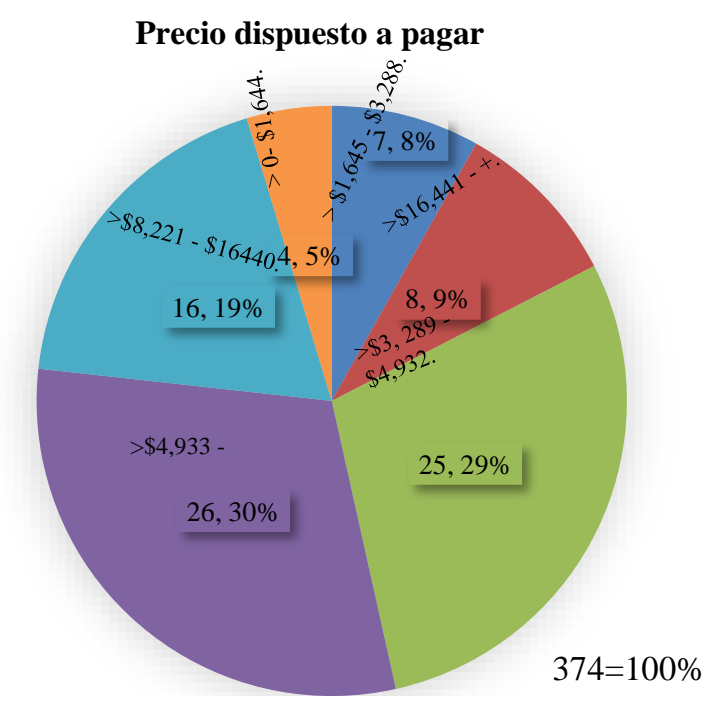

Gráfico 6 Precio dispuesto a pagar

Fuente: elaboración propia; tomada por el estudio: Análisis de estudio de mercado del "Prototipo generador de energía $A K S^{\prime \prime}$

En el Gráfico 6 se presentan las respuestas obtenidas cuando se les pregunto a las personas que cuanto estarían dispuestas a pagar para tener el prototipo.

Los resultados que arrojo fueron que de un $100 \%$, el $26.30 \%$ pagaría $\$ 4,933$, el $25.29 \%$ pagaría $\$ 8,221$, el $16.19 \%$ pagaría $\$ 12,000$, el $7.8 \%$ pagaría $\$ 3,900$, el $4.5 \%$ pagaría $\$ 16,000$.

Estos resultados indican que las personas prefieren el prototipo por el precio más barato, cabe mencionar que son personas que no tienen un amplio conocimiento en la rama de las energías renovables, pero como el rango de precio estuvo muy parejo el porcentaje de aceptación, está bien lanzar el prototipo a un precio elevad ya que debido a que a las personas no les importaría demasiado el costo si de verdad el prototipo ayudara a reducir sus pagos por el costo de luz mes con mes, es por ello que se buscara que el prototipo generador sea de tal manera que se adapte a las distintas necesidades de las personas de la localidad.

\section{Ingresos mensuales en los hogares de Santa Rosalía}

Gallo D. (2017) comenta que, en términos generales, los ingresos son los elementos tanto monetarios como no monetarios que se acumulan y que generan como consecuencia un círculo de consumo-ganancia.
De tal manera que la importancia que estos tienen es que las finanzas de tu negocio deben estar totalmente organizadas y tú como el dueño debes tener claro de cuál es el patrimonio actual de la organización, flujo de caja, nivel de endeudamiento, obligaciones financieras y todo lo demás. También es necesario realizar un análisis de todas estas situaciones es vital para el desarrollo de tu empresa y luego de conocer el estado de las mismas, es cuando se debe ejecutar el plan de negocio para así mejorar los objetivos de cada una de ellas.

Es por ello que los ingresos son una parte fundamental para los habitantes, ya que sin ingresos a sus hogares no podrían tener una vida cómoda y sin preocupaciones, debido a esto se realizó una gráfica, para determinar cuáles son.

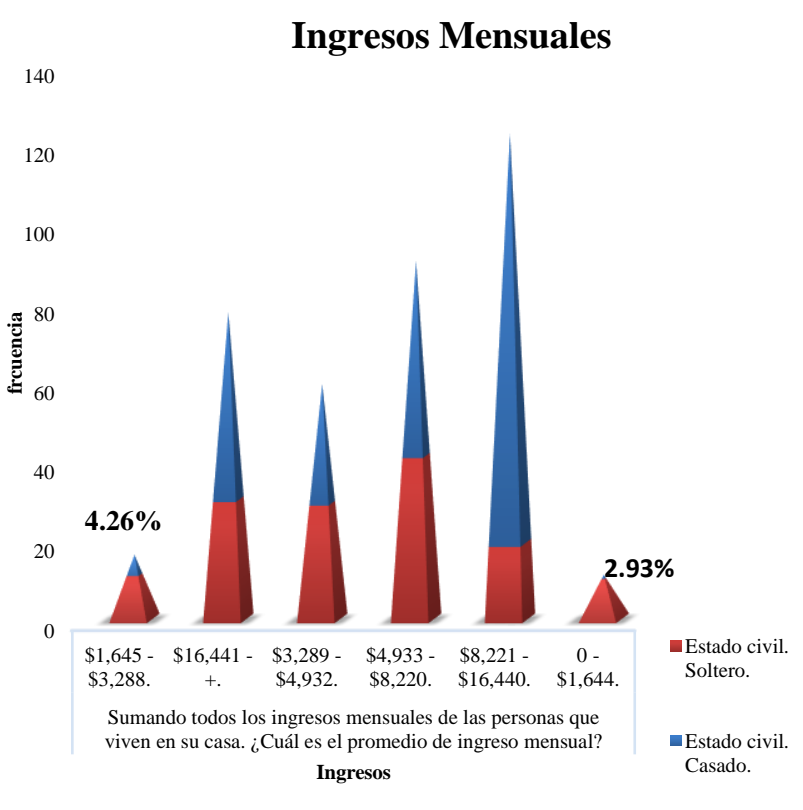

Gráfico 7 Ingresos mensuales

Fuente de elaboración propia; tomada por el estudio: Análisis de estudio de mercado del "Prototipo generador de energía $A K S^{\prime \prime}$

En el Gráfico 7 se puede observar los ingresos mensuales de las personas de Santa Rosalía la cual arroja que un $32.53 \%$ tienen una ganancia de dinero entre $\$ 8,221$ y $\$ 16,440$ y la mayor parte de las personas son casadas por otra parte se observa un mínimo de ingresos con un $2.93 \%$ que es equivalente de 0 a $\$ 1,644$ mensuales los cuales todas las personas solteras. 
El gráfico 8 ayuda a definir que las personas más adecuadas para obtener un prototipo generador de energía son las que obtienen un ingreso de $\$ 8,221$ a $\$ 16,440$ ya que la mayor parte de esta están casadas y pueden obtenerlo fácilmente debido a que no sería una gran disminución en su dinero y no afectaría a su familia por el lado económico. Con ello se determinó que el producto si logrará generar bastantes ventas ya que el porcentaje más alto se obtuvo dentro de los ingresos más elevados, así se dio a conocer que la mayoría de los habitantes cuentan con el recurso económico suficiente como para comprarlo y disfrutar de sus beneficios sin problemas o dificultad alguna para obtenerlo.

\section{Protección del producto}

La protección es una de las cosas más importantes en un producto ya que gracias a él se obtienen distintos beneficios, uno de ellos es que debido a la manera en la que se diseñan logran atraer la atención del consumidor y generar más ventas, el segundo es que teniendo un estilo innovador, atractivo y llamativo se convierte en una competencia grande para los otros productos similares o sustitutos que se encuentran en el mercado, y el siguiente beneficio de este, es la protección, dado que sin él no podría mantenerse en un buen estado. De acuerdo con Alvarado E. (2015) el empaque es primordial para preservar la calidad de los productos en dos momentos clave: el transporte y la comercialización. De modo que es algo que simplemente no se puede dejar pasar, ya que se deben de tomar en cuenta distintas ideas, estrategias y métodos los cuales deben analizarse a fondo, a fin de escoger los mejores para el producto y lograr que todo fluya de buena manera y se obtengan buenos resultados, así mismo cumplir con las expectativas de los clientes.

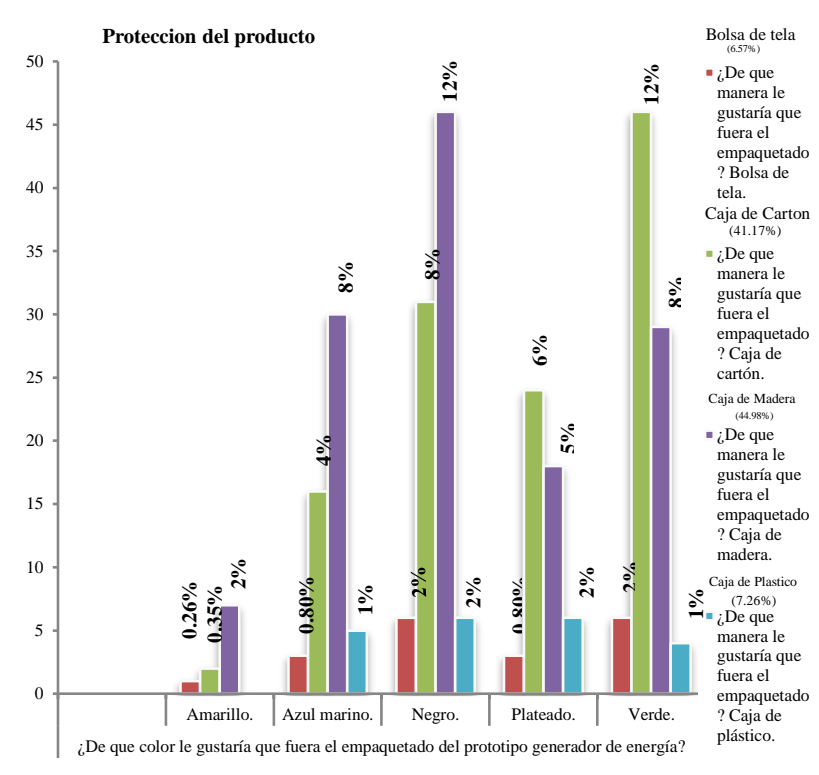

Gráfico 8 Protección del producto; Fuente de elaboración propia; tomada del estudio: Análisis de estudio de mercado del "Prototipo generador de energía

El Gráfico 8 muestra que los resultados obtenidos en el estudio de Mercado realizado informa sobre las preferencias para la atracción de clientes al producto fabricado, como lo es el empaquetado del producto y los colores ya que es una de las partes fundamentales para la atracción de los consumidores, en esta grafica los resultados se muestran de manera cruzada, es decir, comparando las respuestas se arroja a un $6.57 \%$ que los clientes preferirían en que el prototipo se encontrara en una bolsa de tela, el $41.17 \%$ de los consumidores prefieren en caja de cartón, el $44.98 \%$ de los encuestados preferiría en una caja de madera y por ultimo solo el $7.26 \%$ les parecería mejor en una caja de plástico, así pues por el otro lado aparecen diferentes colores con los que los clientes pueden relacionar el empaquetado con su producto, de estas opciones se obtuvieron los resultados de un 5\% en color amarillo, el $16 \%$ de las personas prefieren el color azul marino, también el $16 \%$ esta con el color plateado, mientras que el $31 \%$ de las personas prefieren en color negro aunque por otro lado no se obtuvo respuesta del $32 \%$ de los encuestados.

Con respecto a los resultados se tomó la decisión de crear un empaquetado de cartón y diseñarlo de manera innovadora con el color negro y plateado, con el fin de cumplir con las preferencias de los encuestados, aunque de igual forma se le incluirán otro tipo de colores para hacerlo un poco más llamativo y cumplir con las expectativas de los consumidores. 


\section{Propuesta de mejora para "Prototipo generador de energía AKS"}

De acuerdo con los resultados que se obtuvieron en el estudio de mercado se lograron ver distintos gustos, preferencias y opiniones de los consumidores, los cuales fueron de mucho ayuda, ya que gracias a ellas se pudo mejorar el producto en distintos ámbitos, como por ejemplo: al iniciar con el proyecto el producto no lograba generar la energía suficiente como para hacer funcionar aparatos que necesitan de mucha corriente, como lo podrían ser los abanicos pequeños, licuadoras, planchas, y demás, pero como mencionaban que normalmente se utilizan productos que necesitan gran cantidad de ella, se creó nuevamente el prototipo tomando en cuenta la propuesta anterior y se logró obtener mejores resultados y generar mucho más energía.

Otra propuesta fue mejorar un poco la presentación, dejar de hacerla ver aburrida e incluirle colores más llamativos para lograr llamar la atención de los consumidores fácilmente.

Y uno de los comentarios que mencionaban con más frecuencia fue que se le diera mucha publicidad al producto por distintos medios de comunicación, ya que hoy en día las personas simplemente siguen con la costumbre y no cambian sus servicios o productos tan fácil, de tal manera que mientras no se vea información del producto en redes sociales, carteles u otros medios no se interesarán por él, debido a que es necesario conocer sobre su proceso, beneficios y funciones que contiene para cumplir con las expectativas de los consumidores y lograr que se interesen por su compra.

- Medidor de voltios: Este medidor sirve para saber cuánta carga tiene la pila de 12 voltios.

- Pila de 12 voltios: Esta es la encargada de almacenar la energía generada.

- Inversor de 200w: Tiene la función de sacar la energía almacenada en la pila de 12 voltios y para así utilizarla con los aparatos electrodomésticos.

- Motor generador: Es el que se encarga de generar la energía.

\section{Recomendaciones}

Se sugiere a futuros investigadores que se implemente el uso de hacer una observación directa como la que se llevó a cabo en la investigación, ya que de esta manera se llega a obtener una mayor información que la deseada, esto gracias a que se observa de manera detallada a los encuestados.

También se recomienda aplicar preguntas en la encuesta de manera que las respuestas sean cerradas, como se aplicó en el estudio de mercado, pero siempre y cuando estas sean mejoradas para que así se obtenga una información más precisa y directa.

De igual manera, se aconseja hacer mejoras en lo que viene siendo el logotipo del producto y sobre todo en la protección al producto ya que teniendo un empaquetado que sea llamativo al consumidor es más fácil llamar su atención para generar las ventas deseas del producto.

Asimismo, es recomendable que el proyecto sea llevado a cabo, ya que tiene expectativas altas de introducirse en el mercado y de posicionarse en la mente del consumidor de manera positiva.

Se requiere realizar algunas pruebas de energía para que así el producto se pueda ir mejorando conforme pasen los años y dándole nuevas ideas de estética y mayor capacidad de almacenamiento de energía.

Una vez realizada la investigación anterior se sabe que la propuesta del prototipo generador de energía es una idea considerablemente buena, ya que los resultados obtenidos en el estudio de mercado son muy aceptables por que las personas se inclinan más a utilizar un producto que este realizado con base a las energías renovables que a seguir usando el servicio de energía eléctrica que nos brinda (CFE).

En definitiva, este producto sería de gran ayuda para los habitantes de Santa Rosalía ya que tendría un gran impacto en la localidad, esto se debe a que pocas personas cuentan con algún producto con base a las energías renovables. 


\section{Conclusión}

Actualmente en la comunidad de Santa Rosalía baja california sur existe un gran consumo de energía eléctrica por los habitantes de la localidad, es por ello por lo que existe la necesidad de crear un prototipo generador de energía, para que así ayude a disminuir los pagos por el consumo de esta.

Sin embargo, no existen muchas personas que de verdad quieran utilizar un producto que este hecho con base de las energías renovables, ya que estos podrían ser muy estorbosos o difíciles de utilizar, debido a esto se creó un prototipo generador de energía pensando en las comodidades de las personas y sobre todo que les parezca fácil de utilizar y controlar, por ello se pensó en sacar un provecho más a una bicicleta estacionaria creando un producto que sea capaz de generar su propia energía y al mismo tiempo almacenarla para poder utilizarla en distintos aparatos electrónicos en el momento que sea necesario.

El éxito que se quiere alcanzar con este producto está inclinado a implementar en un futuro las energías renovables, pues si se desarrolla de manera adecuada traerá muchos beneficios, promoviendo la economía y el comercio en la localidad.

Debido a esto se puede decir que el producto tendrá un gran impacto en la comunidad ya que se obtuvieron porcentajes muy altos en la cuestión de las ventas de este prototipo, los cuales arrojan que un $88.44 \%$ estría dispuesto a comprar un producto que su proceso este pensado en implementar las energías renovables en un futuro.

\section{Referencias}

Agencia Internacional de Energía. (18. de febrero. de 2015.). México se une oficialmente a la Agencia Internacional de Energía (AIE) como el $30^{\circ}$ país miembro. gob.mx. Obtenido de https://www.gob.mx.

Alvarado., E. (2015.). El empaque y su influencia en la conducta de compra del consumidor. Quetzal Tenango.
Figueroa., A. (2015.). Analisis del concepto de ingreso desde la teoria contable y economica, que se encuentra presete en la regulacion contable. Universidad Nacional de Colombia. Facultad de ciencias economicas.

Flores., H. (2016). Tesis para optar al titulo de master gestion, sostenibilidad y calidad de las mipymes. Universidad Nacional Autonoma de Nicaragua.Esteli.

Insituto nacional de estadistica y geografia. (2015.). INEGI. Obtenido de https://www.inegi.org.mx/

Mejia., M. N. (2012.). Tecnicas de ventas. Viveros de Asís 96, Col. Viveros de la Loma, Tlalnepantla, C.P. 54080, Estado de México.: Red tercer milenio, S.C.

Ortiz., J. (2015.). La contribucion de la energias renovables al desarrollo, economico, social y ambiental. Universidad de extramadura.España.

Sangri., A. (2014.). Administracion de compras. Primera edicion. Mexico.: Patria.267 pag.

Sweezy., P. A. (2015.). Tesis de la publicidad. Departamento de economia. MacGraw-Hill. Monthly Review. Selecciones en castellano, $3^{\circ}$ epoca, $n^{\circ} 1$. Edicion online. 230 pag.

Villavicencio., J. F. (2016.). Tesis de identificación de preferencias académicas universitarias. Colegio particular Bella Union.Lima- Peru. 119 pag.

Tapia, M. O. (2012.). Interaprendizaje de estadistica basica. Ecuador.: Ibarra.

Gallo, D. (2017). Ingresos monetarios e ingresos no monetarios de los habitantes del casco urbano de puerto Nariño. Universidad Nacional de Colombia. Maestría en estudios Amazónicos. 86 pág. 